\title{
LAS FUERZAS DE LAS NACIONES UNIDAS \\ PARA EL MANTENIMIENTO DE LA PAZ Y LA \\ APLICACION DEL DERECHO INTERNACIONAL \\ HUMANITARIO*
}

\author{
Jean-Luc Blondel
}

\begin{abstract}
La aplicabilidad del Derecho Internacional Humanitario a las Fuerzas de las Naciones Unidas para el Mantenimiento de la Paz surge debido a que éstas, cuyo mandato es mantener la paz en zonas donde existe un conflicto bélico, son armadas. Por esta razón, existen instrumentos jurídicos aplicables a estas situaciones y son los Estados que proveen contingentes los responsables de su difusión, del respeto del Derecho Humanitario y de las infracciones contra él. Más adelante, el autor menciona la opinión del Comité Internacional de la Cruz Roja sobre la aplicabilidad de este derecho y la posición de Naciones Unidas. Se analizan los problemas que se derivan con respecto a las Fuerzas enviadas actualmente a Camboya y la ex-Yugoslavia, especialmente con respecto a la aplicabilidad del Derecho Humanitario y a las actividades del Comité en estas regiones. Por último, se indican las posibilidades de cooperación entre éste y las Fuerzas.
\end{abstract}

\section{Fuerzas de las Naciones Unidas para el Mantenimiento de la Paz: definición y características.}

Las Fuerzas de las Naciones Unidas para el Mantenimiento de la Paz no son ni las fuerzas armadas que el propio Consejo de Seguridad podría constituir en virtud de los artículos 43 y 47 de la Carta, ni aquéllas organizadas por los Estados miembros sobre la base de una invitación (Corea, 1950), o de una autorización (Golfo, 1990) del citado Consejo. Además estas dos fuerzas pueden utilizar las medidas

\footnotetext{
"El presente trabajo utiliza extensamente un estudio interno redactado por el Dr. Umesh Palwankar, miembro de la División Jurídica en la seđe del Comité Internacional de la Cruz. Roja (CICR), en Ginebra.
} 
coercitivas a fin de restablecer la paz y seguridad internacionales en la región respectiva. ${ }^{1}$

Las Fuerzas de las Naciones Unidas para el Mantenimiento de la $\mathrm{Paz}$ difieren de las fuerzas precitadas, primero porque su mandato es de mantener la paz. Dentro de este ámbito, podemos distinguir entre dos grandes tipos de operaciones conducidas por la ONU. Una, consiste en el envío de la misión de observación (ejemplos: UNTSO, Palestina 1948 y UNMOGIP, India y Pakistán, 1949). En este caso, los miembros de la misión sólo tienen la función de observación excluyendo toda posibilidad de jugar un rol activo. Además, no están armados. Por ende, no se trata de fuerzas a las que se les pueda aplicar el Derecho Internacional Humanitario.

El otro gran tipo de operaciones es aquél donde las Fuerzas de las Naciones Unidas tienen el mandato específico de mantener la paz vigilando el cumplimiento de los acuerdos de armisticio o del cese del fuego, que constituyen una condición previa para el despliegue de dichas Fuerzas. En consecuencia, una operación de esta clase es un arreglo temporario con vistas a mantener las condiciones de paz y de seguridad necesarias, específicamente impidiendo o bloqueando el reinicio de las hostilidades para permitir concretar un arreglo de las negociaciones sobre el conflicto en cuestión. La idea es que la presencia física de una fuerza multinacional, neutral e imparcial, tendría sobre los combatientes un importante carácter disuasivo. Dichas Fuerzas de las Naciones Unidas para el Mantenimiento de la Paz fueron constituidas por primera vez en 1956 (UNEP I, Sinaí y Gaza) y continuaron constituyéndose hasta hoy en día (FORPRONU en Yugoslavia, APRONUC en Camboya).

Los contingentes para las Fuerzas de las Naciones Unidas para el Mantenimiento de la $\mathrm{Paz}$ son provistos por los Estados en forma

\footnotetext{
${ }^{1}$ Como obras generales sobre las Fuerzas de las Naciones Unidas ver: R. Higgins, United Nations Peacekeeping 1946-1967: Documents and Commentary, (Oxford: Oxford University Press), Vol I: The Middle East (1969), VoL II:Asia (1970), VoL III: Africa (1980), VoL IV: Europe (1981); F. Seyersted, United Nations Forces in the Law of Peace and War, (Leyden: Sjthoff, 1966); F. T. Liu, "United Nations Peacekeeping Management and Operations", Occasional Paper on Peacekeeping, No4, International Peace Academy, New York, 1990; F. T. Liu, "United Nations Peacekeeping and the Non-Use of Force", Occasional Paper, s.n., International Peace Academy, New York, 1992; S. R. Mills, "The Financing of United Nations Peacekeeping Operations. The Need for a Sound Financial Basis", Occasional Paper, No3, International Peace Academy, New York, 1989; R. E. Lagorio, "Las Naciones Unidas y las operaciones de mantenimiento de la paz en el nuevo escenario internacional", Revista del Ministerio de Relaciones Exteriores y Culto, N21, Instituto del Servicio Exterior de la Nación, República Argentina, Buenos Aires, 1992, pp. 30-42.1
} 
voluntaria, a solicitud del Secretario General de la ONU. Por otra parte, deben prestar su consentimiento el Gobierno a cuyo territorio las Fuerzas de las Naciones Unidas para el Mantenimiento de la Paz deben acudir o las otras Partes directamente implicadas en el conflicto.

Las Fuerzas de las Naciones Unidas para el Mantenimiento de la Paz están bajo el mando de la ONU confiado al Secretario General, pero bajo la autoridad del Consejo de Seguridad, quien está plenamente informado del desarrollo de las operaciones. El comando sobre el terreno es ejercido por un comandante de la Fuerza, nombrado por el Secretario General con el consentimiento del Consejo de Seguridad. El comandante depende del Secretario General.

Sin embargo, una de las características de estas Fuerzas que hace surgir de manera directa la cuestión de la aplicabilidad del Derecho Internacional Humanitario es que estas Fuerzas son armadas. En este sentido, cabe citar el párrafo pertinente del informe que fue hecho por el Secretario General a requerimiento del Consejo de Seguridad en vista del establecimiento, el 19 de marzo de 1978, de la Fuerza temporaria de las Naciones Unidas en el Lỉbano (FINUL): ${ }^{2}$ "D) La Fuerza recibirá armas de carácter defensivo. Ella deberá hacer uso de la fuerza solamente en caso de legítima defensa. La legítima defensa comprendería la resistencia a toda tentativa de impedir por la fuerza el ejercicio de sus funciones, conforme el mandato del Consejo de Seguridad. La Fuerza partirá de la hipótesis de que las Partes en el conflicto tomarán todas las medidas necesarias para asegurar la observāncia de las decisiones del Consejo de Seguridad."

Los problemas teóricos y prácticos ligados a la reạlización de su misión por las Fuerzas de las Naciones Unidas para el Mantenimiento de la Paz dentro de los límites establecidos por el principio rector mencionado precedentemente -sin violar por ello el Derecho Internacional Humanitario- serán examinados más adelante, a la luz de los respectivos mandatos de estas Fuerzas en los casos actuales de Yugoslavia y Camboya. Basta con destacar aquí el riesgo real que encierra el hecho de que, las Fuerzas de las Naciones Unidas sean arrastradas dentro del conflicto, que es lo que ocurrió en la operación en el Congo. En esta situación, las tropas de las Naciones Unidas supieron, en gran medida, reemplazar su mandato por su sola pre-

\footnotetext{
${ }^{2}$ Documento onu s/12611, p. 2.
} 
sencia, por medio de la persuasión y de la conciliación. Pero ellas hicieron igualmente uso de sus armas. De este modo la cuestión de la aplicabilidad del Derecho Internacional Humanitario a las Fuerzas de las Naciones Unidas para el Mantenimiento de la Paz comporta dos aspectos: el respeto que dichas Fuerzas deben tener por el Derecho Internacional Humanitario y la protección de los miembros de las Fuerzas por el Derecho Internacional Humanitario.

\section{Instrumentos jurídicos aplicables a la situación de con- flicto armado.}

Las Fuerzas de las Naciones Unidas para el Mantenimiento de la Paz actúan, evidentemente, en zonas donde se desarrollo o sigue desarrollándose un conflicto armado, internacional o no. De un punto de vista jurídico, los acontecimientos bélicos que qcurren en esa situación están sometidos a reglas internacionales reunidas en el conjunto normativo conocido como el Derecho Internacional Humanitario (o Derecho Internacional aplicable a los conflictos armados). "El Derecho Internacional Humanitario es el cuerpo de normas internacionales, de origen convencional o consuetudinario, específicamente destinado a ser aplicado en los conflictos armados internacionales o no internacionales, y que limita, por razones humanitarias, el derecho de las partes en conflicto a elegir libremente los métodos y los medios utilizados en la guerra, o que protege a las personas y a los bienes afectados, o que pueden estar afectados por el conflicto "3

Definido de esta manera, el Derecho Internacional Humanitario es parte integrante del Derecho Internacional Público positivo, ocupando el lugar del cuerpo de reglas que antes se conocía con la denominación de Derecho de la Guerra. En el estado actual del derecho que rige las relaciones internacionales, después de la prohibición de recurrir a la fuerza en la comunidad internacional, refrendada por la Carta de las Naciones Unidas, los Estados perdieron la

\footnotetext{
${ }^{3}$ Ch. Swinarski, Introducción al Derecho Internacional Humanitario, (Ginebra y San José: Comité Internacional de la Cruz Roja e Instituto Interamericano de Derechos Humanos, 1984), p. 11. Véase también J. Pictet, Desarrollo y Principios del Dexecho Intemacional Humanitario, (Ginebra: Instituto Henry Dunant, 1986); "Derecho Internacional Humanitario", Revista Uruguaya de Derecho Constitucional y Polftico, Serie Congreso y Conferencias, N27, Universidad Católica đel Uruguay "Dámaso Antonio Larrañaga", Facultad đe Derecho, Montevideo, 1992; F. Kalshoven, Corstraints on the Waging of War, (Ginebra: CICR, 1987).
} 
capacidad legal de resolver sus contiendas y litigios por vía de conflicto armado. Quedan todavía sustanciales excepciones a este principio fundamental de la prohibición al recurso de la fuerza. Se admite la legalidad del conflicto bélico en las situaciones siguientes:

- la guerra de legítima defensa, consagrada como el derecho de un Estado de defenderse contra un ataque armado (artículó 51 de la Carta de las Naciones Unidas);

- la guerra de liberación nacional que cumple con las condiciones de un tal enfrentamiento armado en conformidad con las reglas interpretativas de las modalidades de ejercicio de principio de autodeterminación de los pueblos;

- las medidas de seguridad colectiva previstas en los mecanismos del capítulo VII de la Carta de las Naciones Unidas que se pueden tomar en contra de un Estado que represente una amenaza para la paz o para la seguridad internacional.

De todos modos, sea que se lo pretenda conforme o en infracción a las reglas internacionales, todo conflicto bélico continúa igualmente sometido a una normativa internacional. En situaciones en que se usa la fuerza armada, el Derecho Internacional Humanitario tiene dos vertientes que corresponden a sus dos objetivos: el de limitar el recurso a ciertos métodos y medios de combate en las hostilidades, y de proteger a las víctimas del conflicto. En el estado actual, los instrumentos principales del Derecho Internacional Humanitario son los siguientes: 4

- los cuatro Convenios de Ginebra del 12 de agosto de 1949;

- los dos Protocolos Ádicionales a los Convenios de Ginebra del 8 de junio de 1977;

- la Convención sobre prohibiciones o restricciones del empleo de ciertas armas convencionales que puedan considerarse excesivamente nocivas o de efectos indiscriminados, del 10 de octubre de 1980 ;

- la Convención para la protección de los bienes culturales en caso de conflicto armado, del 14 de mayo de 1954.

\footnotetext{
${ }^{4}$ Ch. Swinarski, Principales Nociones e Institutos del Derecho Internacional Humanitario como sistema de protección de la persona humana, (San José: Instituto Interamericano de Derechos Humanos, 1991), segunda edición; Derecho Internacional relativo a la conducción de las hostilidades, Colección de Convenios de La Hayay de algunos otros tratados, (Ginebra: CICR, 1990); D. Schindler y J. Tomán (eds.), The Laws of Amed Conflicts. A Collection of Conventions, Resolutions and other Documents, (Dordrecht y Ginebra: M. Nijhoff Publishers e Instituto Henry Dunant, 1988).
} 
Los Convenios de Ginebra son tratados universalmente reconocidos. Una gran parte de los gobiernos ratificaron también los otros instrumentos relativos a la protección de la persona humana en tiempo de conflicto armado, en particular los dos Protocolos Adicionales a los Convenios de Ginebra. Obviamente, el Derecho Internacional Humanitario debe ser conocido y aplicado. La implementación de ese derecho es una obligación de todo Estado Parte y eso no solamente en el campo de batalla, durante las hostilidades o para proporcionar ayuda a las víctimas de las mismas, sino también en tiempo de paz, como una medida preventiva. Por lo tanto, la difusión de dichos Convenios y Protocolos debe ser parte de la enseñanza militar, de la misma manera que se les enseña a los soldados a manejar una arma. Hay también una responsabilidad internacional de cada Estado para el respeto universal del Derecho Humanitario: en efecto, cada Estado firmante de los Convenios de Ginebra se compromete no solamente a respetar, sino también a hacer respetar el Derecho Internacional Humanitario en todas circunstancias. Como institución internacional encargada de mantener la paz entre los países, la Organización de las Naciones Unidas tiene, por supuesto, un compromiso similar.

\section{Aplicabilidad del Derecho Internacional Humanitario a las Fuerzas de las Naciones Unidas para el Manteni- miento de la Paz.}

La Organización de las Naciones Unidas, como tal, no es parte en los Convenios de Ginebra y no tiene fuerzas propias. Así pues, a los Estados que suministran contingentes incumbe, ante todo, instruir previamente a los miembros de estos contingentes así como deben, por otra parte, instruir a todos los miembros de sus Fuerzas Arma-

\footnotetext{
${ }^{5}$ Véase M. Bothe, "Le đroit đe la guerre et les Nations Unies", Etudes et travaux de l'Institut Universitaire de Hautes Etudes Internacionales, N25, Genève, 1967; Y. Sandoz, "La aplicación del Derecho Humanitario por las Fuerzas Armadas de la Organización de las Naciones Uniđas", Revista Intemacional de la Cruz Roja, N229, pp. 274-284, CICR, Ginebra, septiembreoctubre de 1978 ; D. Schindler, "United Nations Forces and International Humanitarian Law", en: Ch. Swinarski (ed.), Erudes et essais sur le Droit International Humanitaire sur les Principes de La Croix-Rouge, en l'honneur de Jean Pictet, (La Haya y Ginebra: N. Nijhoff y CICR, 1984), pp. 521-530.
} 
das. ${ }^{6}$ Son los responsables de las infracciones contra el Derecho Internacional Humanitario que puedan cometer los soldados que han puesto a disposición y responsables, asimismo, de la represión de esas infracciones.

Los miembros de las Fuerzas de la ONU que sirven durante una operación de mantenimiento de la paz, pueden verse obligados a usar armas (sobre todo en un caso de defensa propia) y quienquiera esté en situación similar debe conocer los principios del Derecho Internacional Humanitario aplicable en los conflictos armados. Al utilizar una arma durante un conflicto e incluso en caso de defensa propia, deben observarse ciertas normas: pensemos simplemente en el respeto a los heridos que ya no combaten.o a los prisioneros tras eventuales enfrentamientos.

No obstante, aunque las Fuerzas de la ONU pueden tener que aplicar directamente el Derecho Humanitario cuando están implicadas accidentalmente en enfrentamientos armados, pueden también desempeñar un cometido importante, colaborando con quienes están especialmente encargados de hacer aplicar ese derecho. Recordemos, en particular, las tareas que incumben a la potencia protectora o a su substituto en territorios ocupados, de conformidad con el IV Convenio. En los lugares donde estén, las Fuerzas de la ONU pueden, por ejemplo, facilitar el movimiento y, en la medida de lo posible, garantizar la seguridad de las personas encargadas de prestar asistencia a las personas civiles aisladas por el conflicto (delegado de la potencia protectora o del Comité Internacional de la Cruz Roja).

En varias ocasiones, el Comité Internacional de la Cruz Roja (CICR) ha afirmado su punto de vista en cuanto a la aplicabilidad del Derecho Internacional Humanitario a las Fuerzas de Mantenimiento de la Paz. En su Memorándum Aplicación y Difusión de los Converios de Ginebra del 10 de noviembre de 1961 dirigido a los Estados Partes en los Convenios y miembros de la ONU, el CrCR llamó la atención del Secretario General de las Naciones Unidas sobre la necesidad de asegurar la aplicación de los Convenios por las Fuerzas puestas a disposición de la Organización. Considerando, como lo hemos visto,

\footnotetext{
${ }^{6}$ Cabe destacar que aunque los soldados que componen las fuerzas de las Naciones Unidas estuviesen específicamente vinculados por los compromisos de sus países de origen; las Naciones Unidas insisten sobre el hecho de que, durante el ejercicio de su mandato, dichas tropas sólo dependen de la competencia propia de la Organización y no de la de sus países de origen.
} 
que las Naciones Unidas no es Parte en los Convenios, el Comité Internacional estima que el Estado que provee un contingente a las Naciones Unidas queda individualmente responsable de la aplicación de los Convenios y, en consecuencia, él deberá hacer lo necesario al respecto, especialmente al dictar las instrucciones, antes de que sus tropas salgan de su territorio. El Memorándum destaca igualmente que, en virtud del artículo primero común a los cuatro Convenios de Ginebra, que compromete a las Altas Partes contratantes también a hacer respetar los Convenios, los Estados proveedores de contingentes estarán de acuerdo en hacer uso de su influencia individual para que las disposiciones del Derecho Humanitario sean aplicadas por el conjunto de los contingentes comprometidos, así como por el comando unificado.

En años anteriores, la XXa Conferencia Internacional de la Cruz Roja (Viena, 1965), en su Resolución XXV ("Aplicación de los Convenios de Ginebra por las Fuerzas de emergencia de las Naciones Unidas") hizo tres recomendaciones que conservan todavía su actualidad:

"La XX" Conferencia Internacional de la Cruz Roja recomienda:

1. que sean concluidos los acuerdos con objeto de asegurar que las Fuerzas Armadas puestas a la disposición de las Naciones Unidas observen las reglas de los Convenios de Ginebra y sean protegidas por ellos;

2. que los gobiernos de los países que proporcionan contingentes a las Naciones Unidas tengan presente la importancia primordial y la necesidad de dar a sus tropas, antes de su salida de sus países de origen, una enseñanza adecuada sobre los Convenios de Ginebra así como la orden de observar estos Convenios;

3. que las autoridades responsables de los contingentes acepten tomar todas las medidas necesarias para prevenir y reprimir las eventuales infracciones a dichos Convenios."

El 10 de abril de 1978, en su nota dirigida al Secretario de las Naciones Unidas, en ocasión de la constitución de las Fuerzas de las Naciones Unidas de Mantenimiento de la Paz para el Líbano (FINUL), el Presidente del Comité Internacional de la Cruz Roja recuerda el contenido del Memorándum y la Resolución ante citados y por una parte se pronuncia sobre las relaciones entre las Fuerzas y el Comité, señalando que ambos llevan a cabo operaciones sobre el mismo terreno, pero en función de diferentes mandatos. Así, "el 
Comité Internacional de la Cruz Roja sabía que podía contar con las Naciones Unidas y su Fuerza de Intervención en el Líbano, para que ellas faciliten allí la libertad de movimiento de sus delegados y garanticen, en la medida de lo posible, su seguridad en las regiones donde el Comité Internacional de la Cruz Roja debe desplegar su actividad humanitaria o convencional".

En virtud de las consideraciones precedentes, el Comité Internacional considera que el mejor modo de compromiso hacia un respeto de los Convenios de Ginebray, si se aplican, de los Protocolos Adicionales, sería que las Naciones Unidas hiciese una declaración de aplicabilidad de los principios y normas del Derecho Internacional Humanitario a sus Fuerzas de Paz cada vez que una de estas fuerzas se constituya. Esta vía podría estar acompañada de acuerdos celebrados entre el Secretario General y los países que proveen los contingentes estipulando la obligación de éstos últimos de asegurar el Derecho Internacional Humanitario por parte de sus hombres (instrucción, sanciones) con la supervisión de la propia ONU. La práctica muestra que esto es lo que las Naciones Unidas ha hecho, a veces, en el pasado.

\section{Posición de las Naciones Unidas.}

En varias ocasiones, las Naciones Unidas reconoció la importancia y la vigencia de las normas del Derecho Internacional Humanitario para las Fuerzas de Mantenimiento de la Paz, aunque no de todas puesto que ciertas normas de los Convenios no pueden aplicarse a las Naciones Unidas o ser aplicadas por ella (por ejemplo aquellas relativas a la ocupación o a la represión de infracciones graves).

Caben mencionarse tres documentos oficiales destacando la posición de las Naciones Unidas:

el Interoffice memorándum dirigido por los señores E. Guyer y B. Urquhart, Subsecretarios generales para asuntos políticos especiales, el 24 de mayor de 1978, a todos los comandantes de las Fuerzas de las Naciones Unidas entonces en ejercicio;

la carta del 23 de octubre de 1978 del Secretario General de la ONU dirigida al Presidente del CICR, en respuesta a la carta de este último del 10 de abril de 1978, en la que destaca que “... los principios de derecho humanitario ... deben, llegado el caso, 
tener aplicación en el marco de operaciones de las Fuerzas de las Naciones Unidas";

- la carta del Secretario General de las Naciones Unidas del 23 de octubre de 1978, dirigida a los Representantes Permanentes de los gobiernos que enviaron contingentes a la FINUL. En ella recuerda que, en situaciones en las que los miembros de las Fuerzas deben usar sus armas defensivas, conforme a la línea directriz $D$ precedentemente mencionada, los principios y el espíritu de las normas del Derecho Internacional Humanitario, “... como están contenidos, inter alia, en los Convenios de Ginebra ... los Protocolos del 8 de junio de 1977 ... deberán aplicarse". $\mathrm{Al}$ efecto, los Estados proveedores de los contingentes deben vigilar que sus tropas conozcan plenamente los principios del Derecho Internacional Humanitario, así como también tomar las medidas necesarias tendientes a asegurar su respeto. En cuanto a la propia ONU, ella “... tendrá a su cargo, a través de la cadena de mando, la tarea de supervisión de que los contingentes de sus fuerzas de mantenimiento de la paz respeten y cumplan con los principios del derecho humanitario".

Ultimamente, en la ocasión de un intercambio de correspondencia entre el Presidente del CrCR y el Secretario General de la ONU, las Naciones Unidas indicó su voluntad de incluir disposiciones relativas a la instrucción y al cumplimiento de las normas del Derecho Internacional Humanitario en los acuerdos con los gobiernos que ponen tropas a disposición de las Naciones Unidas.

\section{Acciones para reforzar la aplicación del Derecho Inter- nacional Humanitario.}

A la luz de todas las observaciones efectuadas precedentemente, se puede proceder a un examen del estado actual de las cosas, en relación en particular a la decisión de las Naciones Unidas de enviar Fuerzas de Mantenimiento de la Paz a la ex-Yugoslavia y Camboya. Sería útil recordar brevemente los puntos esenciales del mandato de las Fuerzas de las Naciones Unidas para el Mantenimiento de la Paz en Yugoslavia (FORPRONU) y en Camboya (APRONUC) relativos por una parte a la aplicabilidad del Derecho Internacional Humanitario 
y, por otra, a las actividades que lleva a cabo el Comité Internacional de la Cruz Roja en estas regiones. ${ }^{7}$

En ambos casos, las Fuerzas de las Naciones Unidas para el Mantenimiento de la Paz tienen componentes de tipo militar, administrativo y de policía. Desde lo "militar", la tarea es de estabilizar la situación en materia de seguridad así como de instaurar un clima de confianza propicio para lograr una solución negociada para la crisis yugoslava y las elecciones en Camboya. Para ello, las Fuerzas de las Naciones Unidas para el Mantenimiento de la Paz deben supervisar el cese del fuego, el retiro de todas las categorías de fuerzas extranjeras de Camboya y de las zonas desmilitarizadas de Yugoslavia, especialmente desarmando y desmovilizando a los combatientes, y protegiendo a las personas que habitan en las zonas desmilitarizadas contra ataques armados erigiendo "checkpoints" en lugares estratégicos. Los miembros de las Fuerzas para el Mantenimiento de la Paz llevan armas ligeras que utilizarán únicamente en caso de legítima defensa, pero también disponen de vehículos armados para el transporte de personal y de apoyo de la aviación.

Lo expuesto permite observar un vasto campo de aplicación del Derecho Internacional Humanitario en lo relativo a las hostilidades, pero sobre todo el conjunto de principios y normas del Derecho Internacional Humanitario relativo a las diferentes categorías de personas protegidas.

El componente civil de las Fuerzas de las Naciones Unidas para el Mantenimiento de la Paz no está armado y tiene como tarea: supervisar de cerca el trabajo de la policía local para garantizar el mantenimiento efectivo e imparcial del orden público y el pleno respeto de los derechos humanos y libertades fundamentales. Para ello, las fuerzas de policía civil acompañan a los miembros de la policía local en sus patrullas y tienen acceso libre e inmediato a todos los locales de ésta o a los que estén bajo su control.

\footnotetext{
${ }^{7}$ Por los detalles de sus mandatos, consultar. Report of the Secretary-General pursuant to Security Council Resolution 721 (1991), S/23280, 11 December 1991, Annex III (para FORPRONU) y Rapport du Secrétarie general (S/2613 et Add. 1), reproducido en Communiqué de presse, Nations Unies, SC/272, 28 fevrier 1992 (para APRONUC). En la fechá de hoy los países siguientes pusieron tropas a disposición de las Naciones Unidas para la FORPRONU: Argentina, Canađá, Jordania, Nepal, Rusia, Bélgica, Luxemburgo, Noruega, Polonia, Dinamarca, Checo-Esiovaquia, Francia, Hungría, Egipto y Ukrania. Para la acción de la APRONUC, 25 países pusieron contingentes a disposición de la ONU, entre ellos: Uruguay, Chile, Malasia, Indonesia, Tailandia, Bangladesh, India, Pakistán, Australia, Países Bajos, Francia, Alemania y Argelia.
} 
El mandato del componente militar de las Fuerzas no hace ninguna mención de su competencia de tomar prisioneros. Sin embargo, el alcance de su mandato deja entrever que en situaciones particularmente tensas, ellas podrían proceder a efectuar arrestos si la situación lo justificara. Esto ya ocurrió en el Congo, y el rol del Comité Internacional de la Cruz Roja en estas circunstancias fue expuesto claramente por un especialista. ${ }^{8}$ En cambio, la fuerza de policía civil de la ONU tiene un mandato específico de supervisar todas las actividades de la policía local y goza de acceso libre a todos sus locales. Esto significa que todos los detenidos por la policía local están también bajo la supervisión de la policía civil de la ONU.

En cuanto a la competencia del Comité Internacional, ella se desarrolla en el mismo terreno que las Fuerzas de las Naciones Unidas para el Mantenimiento de la Paz y es clará en situación de conflicto armado internacional, donde el Comité puede invocar el régimen jurídico del IIII y IV Convenios de Ginebra para poder visitar a los prisioneros de guerra e internados civiles, así como para supervisar su liberación. Sobre la cuestión de la comunicación de los informes de visita, la doctrina del CICR es clara: en general, los informes relativos a visitas efectuadas a prisioneros capturados por las fuerzas de la ONU son remitidas a la potencia de origen, a la ONU y a los gobiernos de los Estados proveedores de los contingentes que capturaron a los prisioneros visitados o de los que los custodian.

Otro aspecto de la aplicabilidad del Derecho Internacional Humanitario a las Fuerzas de las Naciones Unidas para el Mantenimiento de la Paz es, sin duda, el relativo al respeto y a la protección que estas Fuerzas deben al emblema, al personal y a los transportes y unidades sanitarias. En la práctica, tanto anterior como reciente, esto no acarreó problemas. En cambio, en el marco de la difusión del Derecho Internacional Humanitario en los países de afectación de las Fuerzas de las Naciones Unidas, el Comité Internacional de la Cruz Roja podría incitar a las Fuerzas a promover su respeto, parti-

\footnotetext{
8 "Circunstancias desarrolladas de un modo tal que resulta necesario para las Fuerzas para el Mantenimiento de la Paz arrestar y detener a alguno de los mercenarios extranjeros que tomaron las armas contra las Naciones Unidas. Un contingente de los mercenarios fue capturado en un operativo y detenido bajo la custodia de las Naciones Unidas en la base militar de Kamina en el Congo Central. Los detenidos fueron tratados conforme a las normas del III Convenio de Ginebra sobre Prisioneros de Guerra. El trato que las Naciones Unidas brindó a los detenidos fue supervisado por representantes del CICR", en: Bjorn Egge, "Coordinating UN peacekeeping operations and relief and refigee programmes ${ }^{n}$, Paper submitted to the Nordic un Seminar, Oslo, 29 June 1988, p. 13.
} 
cularmente en lo relativo al emblema y a la actividad médica del Comité cerca de las unidades armadas de las partes en el conflicto.

Se trata de identificar las lagunas y problemas (existentes o previsibles) tanto en el plano general (CICR - ONU - Estados proveedores de los contingentes) como en el plano operacional, a saber: la aplicación y el respeto del Derecho Internacional Humanitario por las propias Fuerzas de las Naciones Unidas, el rol de éstas para contribuir al respeto del Derecho Internacional Humanitario por las distintas entidades en el lugar, y la cooperación entre las Fuerzas y el Comité, así como también pensar en los trámites y diligencias que se podrían iniciar.

La responsabilidad principal sobre el plan operacional de asegurar la aplicación y el respeto del Derecho Internacional Humanitario por las Fuerzas de las Naciones Unidas incumbe a las Naciones Unidas. Sin embargo, el Comité Internacional de la Cruz Roja está listo para ayudar al Secretario General y las Fuerzas contribuyendo, dentro de sus posibilidades, por ejemplo proveyendo un marco de instrucciones en Derecho Internacional Humanitario que podría ser adaptado a las necesidades específicas de cada Fuerza.

\section{Cooperación entre las Fuerzas de las Naciones Unidas para el Mantenimiento de la Paz y el Comité Internacio- nal de la Cruz Roja.}

Respecto de las posibilidades de cooperación y coordinación entre las actividades humanitarias de las Fuerzas de las Naciones Unidas y del Comité, la práctica, hasta el presente, indica que deberían considerarse los siguientes puntos: búsqueda de personas; información mutua; seguridad del personal del Comité; investigaciones en caso de violación de los Convenios de Ginebra o de otro tratado; visita, liberación o repatriación de prisioneros de guerra; evacuaciones médicas; transporte; servicios sanitarios. A título de ejemplo, podrían citarse los siguientes casos ocurridos en el pasado:

- en su informe relativo a las operaciones de la UNFICYP (en Chipre), el Secretario General precisó: "con respecto a las actividades humanitarias se ha realizado una reunión ... con representantes del Comité Internacional de la Cruz Roja y hubo acuerdo en que la Cruz Roja debería asumir la responsabilidad 
primaria para proveer asistencia y socorros a los refugiados, prisioneros, personas desaparecidas, alegación de atrocidades y problemas similares que están, tradicionalmente, dentro de la competencia del Comité Internacional de la Cruz Roja. UNICYP deberá continuar jugando su rol activo, el cual incluirá la conducción de investigaciones y negociaciones locales y asistirá y cooperará en la conducción de las operaciones de ayuda humanitaria ..."; 9

- en una Resolución de la Asamblea General, ésta demandó al Secretario General “... proveer sus buenos oficios a través de su Representante Especial en Chipre, a fin de apoyar el establecimiento de un cuerpo de investigación con la participación del Comité Internacional de la Cruz Roja, el cual debería poder funcionar en forma imparcial, efectiva y rápida ..."; 10

- en el contexto de las operaciones de la ONU en el Congo (ONUC) una nota del representante especial del Secretario General destacaba: "EL Secretario General ... (reitera) su pedido de que se permita otra vez a las Naciones Unidas, por medio de representantes de la Cruz Roja, visitar personas detenidas y comprobar el trato que se les dispensa". 11

Actualmente, en Camboya, aun cuando la repatriación es competencia principal del Alto Comisionado de las Naciones Unidas para los Refugiados, toda la infraestructura médica y quirúrgica del Comité Internacional de la Cruz Roja está, evidentemente, puesta a disposición de las personas repatriadas. Por otra parte, el CICR emprende esfuerzos particulares en el campo de la Agencia dentro de las zonas controladas por las distintas facciones. '?

Al igual que en Camboya, el Comité colabora de manera muy estrecha con los representantes del Secretario General o de los organismos dependientes del sistema de las Naciones Unidas en la ex-Yugoslavia (en particular con el Alto Comisionado de las Naciones Unidas para los Refugiados), en Somalia (en particular con el Programa Alimenticio Mundial) y en otras situaciones similares.

Para cumplir con la intención de concretar esta cooperación humanitaria es necesario que el Comité Internacional de la Cruz Roja continúe hásta identificar los ámbitos propicios y hasta concre-

\footnotetext{
${ }^{9}$ S/11353/Add. 12, 31 de julio de 1974, párrafo 5.

${ }^{10}$ Resolución AG 32/128, 16 đe điciembre de 1977.

${ }^{11} \mathrm{~S} / 4590,9$ de điciembre de 1960 , párrafo (f).
} 
tar los "working agreements" con los comandos de las Fuerzas de las Naciones Unidas para el Mantenimiento de la Paz. En cuanto a la cooperación con las Fuerzas Armadas, es sobre todo en el campo de la difusión del Derecho Internacional Humanitario que se podría ampliar el apoyo del Comité, por ejemplo llevando a cabolas acciones puntuales siguientes:

- difusión para los contingentes militares antes de su partida, efectuada por cada delegación del CICR;

- informe de los comandantes de los contingentes en ocasión de su paso por la sede de las Naciones Unidas en New York, por la delegación del CICR ante la ONU en dicha ciudad;

- difusión en los países de afectación efectuada por los delegados presentes o por especialistas de la difusión a las Fuerzas Armadas.

Durante los seminarios y conferencias dictados a las Fuerzas Armadas debería ponerse el acento en que la aplicabilidad y el respeto del Derecho Internacional Humanitario son del interés mismo de los miembros de las Fuerzas de las Naciones Unidas para e] Mantenimiento de la Paz que, en los casos concretos, no tienen el beneficio de todo un sistema de protección y responsabilidad resultante de los instrumentos del Derecho Internacional Humanitario. Hasta se podría dar un paso más y considerar la difusión de los principios y normas fundamentales del Derecho Internacional $\mathrm{Hu}-$ manitario por las propias Fuerzas hacia los miembros de las unidades armadas de las partes en conflicto, con quien éstas podrían tomar contacto. Esto se inscribiría en el marco de responsabilidades de las Fuerzas como una consecuencia de la aplicación del Derecho Internacional Humanitario (obligación de contribuir a hacer respetar ese derecho). Las instancias concretas a seguir serían las de identificar o, en cada caso alentar, la designación de un responsable de los asuntos humanitarios en el seno de las Fuerzas de las Naciones Unidas, a fin de crear un grupo de oficiales quienes podrían enseñar el Derecho Internacional Humanitario, tanto en el seno de estas Fuerzas como en el de las Fuerzas Armadas de la región respectiva. 